\section{ESTUDIOS SOBRE LA DEFENSA NACIONAL EN LAS UNIVERSIDADES PÚBLICAS Y PRIVADAS: LA PERSPECTIVA DE LA UNIVERSIDAD CEU-SAN PABLO}

\author{
Leopoldo Seijas Candelas \\ Profesor Agregado Universidad CEU-San Pablo
}

\section{NATIONAL DEFENCE STUDIES IN THE PUBLIC AND PRIVATE UNIVERSITIES: THE PERSPECTIVE OF THE SAN PABLO CEU UNIVERSITY}

\begin{abstract}
The studies regarding the National Security and Defence within the Spanish university have been recently implemented. It has been certainly difficult to achieve that this concepts become mentioned in the lessons and that they contribute to create the called "Defence culture". The university cannot ignore the reality of our society and the actual turbulent situations we are facing since the university should be the reflection and projection of the whole society. According to this, the San Pablo CEU University, aware of the importance of this knowledge area, has included this subject within Information Sciences studies.
\end{abstract}

KEY WORDS: Security; Defence; university; Information Science; knowledge; society.

\section{UNIVERSIDAD Y SOCIEDAD}

Antes de iniciar nuestro trabajo, se hace necesario aclarar que el término "sociedad" lo vamos a utilizar aquí en su sentido más estricto de sociedad civil, sin considerar como parte de ella al Estado. Es decir, nos referimos a la sociedad en cuanto fenómeno social pero no como organización política. Es obvio que la importancia y la especialidad de los problemas en las relaciones entre la Universidad privada y el Estado merecería, en su caso, un trabajo de otra índole.

Nos plantearemos los problemas alrededor de dos interrogantes fundamentales: Qué puede esperar la Universidad de la sociedad y qué puede esperar la sociedad de la Universidad. Nos referimos a las Universidades privadas que en lenguaje jurídico español serian tanto las que se crean al amparo de la nueva regulación constitucional como las creadas o por crear bajo la cobertura de las relaciones Iglesia-Estado.
RESUMEN: Los estudios sobre la seguridad y la defensa, en el ámbito universitario español, son de reciente implantación. Ha sido difícil que estos dos conceptos entren en las aulas y que contribuyan a lo que se conoce como "cultura de la defensa". La universidad no puede ir contra la realidad de la sociedad y de las convulsiones de los tiempos actuales, porque en definitiva, ésta es reflejo y proyección de la sociedad total. En este sentido. la Universidad CEU-San Pablo, consciente de la importancia de esta área de conocimiento incorporó esta materia en sus planes de estudios en la carrera de Ciencias de la Información.

PALABRAS CLAVE: Seguridad; Defensa; Universidad; Ciencias de la Información; conocimiento; sociedad.

\section{EXPECTATIVAS UNIVERSITARIAS RESPECTO DE LA SOCIEDAD}

Ante todo, la Universidad tiene que recibir de la sociedad tres elementos fundamentales: el profesorado, el alumnado y los recursos financieros.

Respecto de los alumnos, la Universidad debe mantener dos principios como derecho propio: el derecho a tener su ideario (lo cual reza naturalmente con los profesores) y el derecho a seleccionarlos con criterios objetivos. Inmediatamente aparece la cuestión de los becarios como medio de incorporación de alumnos capacitados carentes de medios económicos para acudir a las universidades privadas. Sin perjuicio de la existencia de tales becas, nuestra posición es, en lo esencial, muy diferente. Lo más importante es hacer un esfuerzo de racionalización de costes que sitúe el nivel de cuotas lo más bajo posible, haciendo asi de la baratura de la universidad, sin mengua 
de su calidad, una característica fundamental que impida el cierre al alumnado de las clases medias, en el sentido amplio de este concepto.

Estamos asistiendo a una floración de iniciativas de la sociedad civil en torno de la investigación y a la educación que alcanzan incluso la promoción y desarrollo de universidades privadas. Nos estamos refiriendo, como es lógico, al desarrollo y puesta en marcha del llamado Espacio Europeo de Estudios Superiores, más conocido por el "proceso de Bolonia".

Muchas de estas iniciativas se vienen canalizando a través de fundaciones que buscan inspiración en la experiencia y tradición de las grandes fundaciones americanas. Se trata de fundaciones que han dejado de ser instrumento al servicio de los intereses de las empresas o grandes empresarios que las crearon, para centrarse en actividades de evidente interés colectivo y de efectos beneficiosos para el desarrollo de la sociedad civil.

En este punto y sin desdeñar en absoluto las aportaciones permanentes y sin un destino específico de determinadas instituciones sociales, es claro que la financiación privada se canaliza a través de programas concretos de acción universitaria ya sea de docencia o de investigación.

Encontramos asi fundaciones y otras instituciones que no encuentran fácil su acomodo en el desarrollo de su capacidad de filantropia, altruismo y servicio a la sociedad y que están deseosas de participar en programas universitarios atractivos que les permita diferenciarse de los modelos de fundaciones existentes. Es aquí, en esta colaboración, donde la universidad ha de tratar de conseguir la estimación y respeto de la sociedad, demostrando en su trabajo su credibilidad a través de la obra bien hecha, ganando la mejor reputación a largo plazo y poniendo a prueba que sabe desarrollar sus actividades en una sociedad libre en plena competencia con un Estado acostumbrado a monopolizar el campo de la enseñanza e investigación.

Pero no se trata sólo de atraer financiación privada para programas concretos. No estará de más recomendar a las instituciones beneficiarias de las aportaciones privadas, de la necesidad de contar con la opinión de los mecenas, de informar periódicamente de las actividades desarrolladas y de rendir cuentas de los trabajos realizados y del destino de los fondos recibidos.

En punto a los recursos y sin desdeñar en absoluto las aportaciones permanentes e indiscriminadas de determinadas instituciones sociales, se tiene que hacer una oferta de rigurosa seriedad: la financiación privada ha de recaer sustancialmente sobre programas concretos de acción universitaria, ya sean de docencia o de investigación. No estará de más recomendar que a los posibles mecenas se les tenga siempre bien informados y se recabe su opinión. No hay mayor causa de rechazo ante quienes han de ejercer un mecenazgo de cualquier naturaleza que el que se les llame "para pedir dinero" y se les ignore a la hora de pedir opiniones. Hemos de pensar que grandes fundaciones nacionales y extranjeras se van retirando de las subvenciones sin finalidad concreta.

La universidad privada tiene que buscar y promover en el seno de la sociedad fuerzas organizadas, ya sean políticas o profesionales, que le presten el apoyo sociopolítico necesario frente a los intentos estatificadores que no cesarán nunca.

\section{Qué puede esperar la sociedad de la universidad}

En principio, expertos competentes en las distintas ramas del saber que de una manera muy directa afectan a la vida social, como son los trabajadores universitarios del mundo empresarial y los médicos. Hay que dejar bien claro desde el principio que las carreras que no tienen esta connotación de una manera clara y abierta (como las de Ciencias básicas y las estrictamente humanísticas) han de ser impartidas para el pleno cumplimiento de la misión intelectual de la universidad. Muchos acontecimientos de estos últimos tiempos son argumentos a favor de esta tesis. En primer lugar, porque sin ciencia básica no hay tecnología adecuada $y$, en segundo lugar, porque los conocimientos humanísticos cada vez tienen un papel más relevante en los problemas de organización social y en los que la "cultura" se ha convertido afortunadamente en una exigencia de la sociedad moderna.

En términos generales puede anticiparse que la sociedad espera de la universidad algo muy específico y difícil de conseguir: "calidad" que puede contemplarse desde una doble vertiente: calidad del profesorado y calidad de la 
enseñanza, resultado evidente que sólo una universidad dirigida por un profesorado de calidad puede prestar los servicios de alto nivel que la sociedad demanda. Por tanto, en primer lugar, la sociedad requiere del profesorado una serie de cualidades, una serie de condiciones necesarias para poder depositar su confianza en la universidad y que podrian concretarse en dedicación, preparación, competencia y permanente actualización.

En segundo lugar, y como consecuencia de lo anterior, la sociedad espera que la universidad imparta una enseñanza de calidad, entendida en un sentido amplio y no limitado estrictamente al estudio de unas asignaturas determinadas.

En una clasificación integradora de las múltiples actividades que se producen en la universidad y que, como parte indisoluble de la comunidad, afectan a la sociedad civil, son dos las que habitualmente se configuran como constitutivas de su acción específica: la función docente y la investigadora.

Las dos son imprescindibles para el desarrollo de la sociedad en su conjunto y en ambas tienen un compromiso de corresponsabilidad tanto la propia sociedad como los profesores y alumnos que, en sentido estricto, constituyen la comunidad universitaria.

El profesor deberá investigar, enseñar y formar estudiantes, entendiendo el concepto de estudiantes en el sentido orteguiano superador del concepto de "alumno".

Destacaba Ortega y Gasset, al iniciar un curso de metafisica, que estudiante sería solamente aquel para quien el conocimiento de una disciplina constituyera una necesidad, no una condición para superar unos exámenes, sino una necesidad tal que le llevará a continuar investigando; un deseo de saber que sintiera en los más profundo de su naturaleza y por el que continuaría la labor del maestro para intentar crear y descubrir verdades nuevas.

Hermosa e ilusionante sin duda esta visión, investigadora y docente, del profesor universitario pero en la que, dada la imperfección humana y las diferentes capacidades y aptitudes de los hombres, puede prevalecer una u otra, sin que ello represente carencia total de cualquiera de ellas.
La sociedad espera de la universidad profesionales aptos, desde el punto de vista profesional, para el desempeño de sus actividades, pero, y quizás con mayor trascendencia, para aspirar al bien común como idea irrenunciable: hombres y mujeres sólidamente formados con criterios y comportamientos éticos y morales.

Para conseguir estos resultados son múltiples las capacidades que deben concurrir en un profesor universitario que trata de realizar con la máxima eficacia su función docente.

Sin pretensiones de exhaustividad podrían mencionarse las siguientes:

1. Un amplio y seguro conocimiento de su disciplina que deberá ser constantemente actualizado, procurándose la información necesaria de las últimas aportaciones sobre la misma: textos, revistas, comunicaciones, etc. (esta actividad tiene un componente investigador que resulta imprescindible como medio, si bien no como finalidad específica para quien con mayor dedicación se ocupa de la docencia).

2. Información, previa y lo más amplia posible, de los conocimientos de los alumnos respecto a todas las disciplinas y especialmente de las relacionadas con la propia materia, para adecuar las explicaciones a la capacidad de entendimiento de los estudiantes. Resulta ineficaz y decepcionante, en especial para los alumnos que el profesor dé por conocidos, por el simple hecho de haber superado un examen anterior, aquellos principios o conocimientos necesarios para entender con facilidad las explicaciones de la nueva disciplina.

3. Capacidad pedagógica suficiente que permita desarrollar, con sencillez pero sin pérdida de rigor, las explicaciones que permitan una fácil comprensión por los alumnos y fomenten su interés por el resto de la disciplina. En este sentido la experiencia docente ha demostrado la utilidad de iniciar los cursos, a modo de introducción, con una síntesis general del contenido total de la disciplina, a fin de que el alumno conozca desde el principio del curso la dinámica del mismo y tenga una visión global de la materia, que se irá desarrollando en profundidad a lo largo del programa. Esto podría facilitar la comprensión de las interrelaciones estructurales de todos y cada uno de los capítulos y su conexión con otras disciplinas. 
4. Por último, y en tanto persistan las actuales estructuras educativas, es una necesidad imprescindible como insatisfactoria, la existencia de exámenes para juzgar el grado y la suficiencia de los conocimientos adquiridos por los alumnos.

Se aplican diversas modalidades de examen siendo las más frecuentes unas pruebas colectivas escritas, mediante dos o tres convocatorias, dependiendo si se trata de una asignatura anual o cuatrimestral.

En este punto es exigible al profesor, como mínimo inexcusable, un riguroso criterio de equidad y justicia en la apreciación y calificación de los saberes adquiridos.

Este mínimo exigible, justicia y equidad, será tanto más factible de alcanzar para el profesor cuanto mayor sea el conocimiento personal e individualizado de sus alumnos.

No se nos oculta que lo anterior exige, al menos, la concurrencia de dos premisas:

a) Un número prudente de alumnos por grupo, lejos de las desmesuradas cifras que, con más frecuencia de la deseable, figuran en las aulas de la universidad pública.

b) Una mayor dedicación del profesorado, tanto en el aspecto temporal como en el personal, con espacios de convivencia con los estudiantes, superando la práctica habitual de una mera, aunque asidua y puntual, asistencia a clase durante las horas lectivas (con este propósito es conveniente, para evitar la ausencia del alumno al terminar su horario de clases, intercalar algún período de descanso que le permitiera conversar con los profesores sobre aquellas cuestiones que le interesaran o inquietaran).

Con estas premisas podría alcanzarse, quizás no de manera inmediata pero sí a medio plazo, una superación de los exámenes en su forma actual y se evitaría el riesgo de una calificación inevitablemente aleatoria.

\section{La construcción actual de la multiversidad}

Las sociedades determinan y organizan en cada momento concreto de su historia el tipo de educación ${ }^{1}$ adecuado a su mantenimiento y reproducción. Ese determinado tipo de educación está en función, de las concepciones ideológicas, de las posiciones existenciales, de las cosmovisiones y de necesidades de autoconservación e intercambio de carácter económico. Para decir lo que quiero decir, seguiré pues, el paradigma -representado más genuinamente que nadie por Veblen-que interpreta el papel de la educación en cuanto condición de la estructura y el cambio social. El saber que transmite la educación no puede ser neutro, sino plenamente interesado: es producto de las estructuras sociales, y al tiempo, constituye un constante refuerzo de ellas mismas. De modo que los saberes que presentan jerarquizados y estratificados en razón de la función que se les atribuya.

La educación es uno de los instrumentos centrales de control social. El sistema educativo mantiene como función primordial el logro de un mínimo de conformidad social $\sin$ el que ninguna sociedad podria subsistir. Todas las instituciones y prácticas educativas viven en consonancia con las restantes instituciones sociales y están sujetas a las mismas reglas de evolución al interno de la estructura global de la sociedad en la que se realizan. Solamente conociendo de la sociedad global es posible conocer y comprender el microcosmos educativo. Vivimos en una sociedad civil fuertemente debilitada. $Y$ no puede predicarse nada distinto de nuestro sistema educativo.

Nos estamos refiriendo, en este trabajo, a la universidad y no queda más remedio que de contemplar el todo en su condición de enfermo, hablando de la universidad hemos de referirnos a un estadio patológico de suma gravedad, mucho más cuando la sociedad total concibe la enseñanza universitaria como un servicio público y/o privado que se dirige a clientes que deben quedar satisfechos. Y conviene no olvidar que el cliente siempre tiene razón. No es hoy la universidad, precisamente, una comunidad intelectual autónoma, un centro crítico, un foro de encuentro de pensamientos independientes buscando la verdad. Pero la opinión pública no parece entrañar esas cualidades. De manera que cada vez sean más los estudiantes que se interesen por la universidad únicamente como escuela profesional de tránsito obligado hacia el mercado de las ocupaciones. A pocos importa, dentro o fuera de la institución académica, que esa enseñanza "profesionalizada" que se demanda lleva a la "producción" de sujetos dóciles, sin apenas capacidad crítica. Asistimos a una rectificación creciente de la enseñanza universitaria. 
Pero no parece tener mucho sentido resucitar el infructuoso debate de fines del s. XIX entre humanismo y técnica, ni tampoco el de hace casi cincuenta años a propósito del espacialismo y la educación liberal. Sea como fuere, nada obliga a la aceptación pacífica de las consecuencias que pueda traer consigo el empeño en la ignorancia de la responsabilidad social de la institución universitaria, gravemente lesionada ante el empuje de "la interiorización de la lógica burocrática"2. Esa misma lógica, denuncia Vallespín, se hace visible en el consenso generalizado respecto de la primacía del interés corporativo, por cierto, puesto de manifiesto, una vez más, con ocasión de las últimas reformas de los planes de estudios de ciertas carreras como "otra muestra más de esa pérdida de la visión globalizadora de la universidad actual". Lo más grave sigue siendo, sin embargo como bien ha señalado Carlos García Gual, el proceso de especialización de las facultades, la insistencia en las técnicas profesionales, la obtención de un conocimiento puramente instrumental que no hace sino ahondar en la pérdida del hilo conductor unitario guiado por la visión crítica ${ }^{3}$.

El sistema educativo señala el orden taxonómico de los diversos bienes culturales y los distribuye, en tanto capacita y sitúa a los individuos según las exigencias y necesidades sociales, con lo que viene a desempeñar la función nuclear de armonización y cimentación de las estructuras sociales, políticas y económicas. La educación es, pues, ante todo, un factor de legitimación y dominación. Con todo, quien no acepte esta tesis, podrá hacer suya sin mayores problemas la visión de Parsons respecto de la doble función de las instituciones educativas. Por un lado, "socialización": "el hecho de desarrollar en los individuos los compromisos y aptitudes que son requisito indispensable del desempeño de su rol de futuro". Por otro, "selección": "asignación de recursos humanos dentro de la estructura de roles de la sociedad adulta"4.

Sea cual fuere la posición adoptada, no es posible desconocer el imperativo histórico que ordena que la educación se someta a las exigencias sociales en tanto la legitimidad del saber aparezca impuesta por la resultante de las complejas relaciones entre el mismo saber y las posiciones sociales de poder. Si las metas sociales requieren técnicos, burócratas y tecnócratas, las instituciones educativas deberán hacer provisión de tales individuos, o dicho con otras palabras, no parece abrirse otra salida a la educación superior que la sumisión a las fuerzas racionalizadoras de una cultura dominada por la técnica. En cualquier caso no se trata de un fenómeno nuevo.

Si la Universidad de Berlín, que ya en los primeros años del s. XIX marcó pautas de modernización seguidas en buena parte por tantas otras universidades europeas y americanas, tenía como primer objetivo el desarrollo del saber, y en segundo lugar, y tal vez como una concesión, el preparar a las clases de profesiones liberales y dirigentes, pudo sostener Saint-Simon, que la educación, sin perjuicio de su propósito de formación integral, había de presentar una finalidad utilitarista, vale decir, la mejora de la sociedad del trabajo y la productividad, orden de designios propio de una sociedad industrial que tenía que cuajar como tal. Más de siglo y medio después, hemos de constatar con tristeza que es el saber empírico el que preside el sentido de nuestros fines educativos y que las controversias berlinesas siguen vigentes.

Compete a la universidad la consagración de los cuadros superiores y rectores de la sociedad. Al tiempo, se configura la institución universitaria como uno de los principales factores de legitimación del orden establecido a través de la formación del consenso social. Por ello, no puede dejar de contemplarse a nuestra institución como gran correa de transmisión de las ideologías dominantes.

No sorprenda, pues, que siga reprochándose a la universidad la deficiente adaptación de sus enseñanzas a las necesidades de las empresas y el mercado laboral. De manera que es ya un lugar común la percepción de que la enseñanza superior y la evolución de los empleos profesionales suelen marchar a distinto paso. Y la endeble conexión entre la universidad y la empresa queda más visible en momentos de agudeza de las crisis de mercado, de los cambios tecnológicos y sus repercusiones en la movilidad de las ofertas de empleo, circunstancias, por cierto, que no hacen más que complicar, aún más, las necesarias previsiones sobre las readaptaciones profesionales en el decurso de las exigencias del mercado y la oferta polivalente de la universidad.

Tal inadecuación al sistema laboral y ocupacional ha de verse acrecentada, más si cabe, por las referidas reformas que, a causa de una cierta confluencia de intereses - de orden corporativo, sobre todo-, configuran, hasta lo que 
conocemos, programas de estudio tanto o más obsoletos, rígidos, mediocres, faltos de sentido y poco interdisciplinarios como aquellos que han pretendido mejorar.

La universidad no puede ir contra la realidad de la sociedad contemporánea. Y, a crear de ello, la preocupación esencial de los que controlan los recursos educativos no parece sino encaminada a la formación de productores y consumidores, dejándose llevar por una mera apariencia de realidad que empieza a quebrarse incluso en los reductos más interesados en su vigencia. Correlato de la obsesión por "formar" y "profesionales" vendría a ser, entre otras, la evaporación del valor del conocimiento "puro" y hasta del sentido no instrumental del saber. No pueda extrañar, entonces, el continuo crecimiento del número de personas y proyectos ignorantes de las responsabilidades sociales, morales o incluso epistemológicas. Escribía Vallespín comentando el libro de Allan Bloom (El cierre de la mente moderna, Plaza-Janés, Berna, 1989) sobre el estado actual de la universidad americana: "La crisis espiritual que afecta a la sociedad revierte directamente sobre la universidad...." La universidad va haciendo suya la lógica del proceso productivo y técnico-administrativo de manera que aparece "colonizada por las nuevas leyes que aseguran su enganche instrumental a los nuevos fines" 5 . $Y$ añade el profesor Vallespín: "El resultado es la universidad de masas", en el sentido de Tocqueville.

$Y$ en efecto, se ha instalado en nuestras sociedades un penetrante ignalitarismo, que como advirtiera Tocqueville, alimenta, en aparente paradoja, al individualismo y al materialismo. La vida monótona y unitaria, la de la opinión pública, amenaza, "desde abajo", la libertad y la autonomía del sable. "La minoria humana fue obra de leyes y no de la providencia", puede sostener al autor de La democracia en América.

Cierto es que a una sociedad pluralista ha de corresponder una universidad pluralista, una "multiversidad". La universidad es reflejo y proyección a la vez de la sociedad total $y$, por tanto, el espacio en que la sociedad renueva de continuo las condiciones sociales de su propia existencia. Pero "también es cierto que en la sociedad pluralista los asuntos comunes se determinan con relativa facilidad porque como observa Thomas Ellwein, se aproximan al punto cero de los valores" ${ }^{6}$. Hasta el mismo D. Riesman ha llegado a señalar enfáticamente el hecho de que sean tan pocos los profesores que muestren hoy interés en moldear lo que "sólo raramente recibe la denominación de carácter o conciencia moral de los estudiantes y enredarse en discusiones con los jóvenes sobre principios en los que los mismos educadores sólo creen a medias". Y he aquí la conclusión de Habermas al respecto: "Puesto que los estudiantes se aferran a la máxima de la formación a través únicamente de la ciencia, pero interpretan la función de esta ciencia de modo positivista, limitándola al cuerpo de la disposición técnica, se ven formados a expulsar del templo de la enseñanza superior 'la formación intelectual general' y la 'configuración armónica de la personalidad.' Con ello no se hace más que separar una vez más del proceso formativo del conocimiento un elemento, éticamente solidificado, por así decir, de la formación del carácter, un elemento al que otros sólo pretenden recibir a las puertas de la Universidad para conducirlo a través de las dependencias de la administración universitaria"7.

¿Qué salidas cabe proponer ante esta situación? Sin perjuicio de otras consideraciones que podamos hacer a lo largo de este trabajo, quiero formular ahora algo que no debería quedarse en mero desideratum: la universidad ha de afirmarse en la responsabilidad de la formación integral de los estudiantes y de los profesores, entre otras razones, también, porque no se puede arrojar la toalla ante la exigencia de una constante elevación de la calidad de la producción intelectual de los universitarios y, por consiguiente, de su actividad profesional.

La universidad se vincula, cada vez más estrechamente, con la organización económica de la sociedad. La comprensión de tal conexión permite la explicación de las funciones sociales de la institución académica. En las sociedades fuertemente industrializadas, administradas en términos de racionalidad burocrática, los sistemas educativos tienden hacia la preeminencia de la preparación especializada en el orden técnico y minimizan la formación integral. Existe hoy una identificación mecánica entre la especialización y técnica, de modo que la educación liberal - o generalista o integral, términos que, aunque no puedan reputarse sinónimos expresan una línea de concepciones-, se correspondería, en lo esencial, con la enseñanza de materias humanísticas. Asistimos, pues, a una tendencia social extraordinariamente poderosa: el especialista ha desplazado con rotundidad a aquel hombre que hacía del saber global y fundamentado, en constante revisión, la 
experiencia suprema. Incluso puede decirse que la opinión pública tiende a identificar al "hombre cultivado" con la figura del diletante.

$Y$ es que las necesidades y los proyectos sociales van de la mano de la historia. Weber nos enseñó que la figura del "hombre cultivado" primó sobre la del "especialista" y sentó las bases del prestigio social en sistemas tan dispares como las estructuras de dominio feudales de carácter teocrático y patrimonial.

Pero "el descubrimiento (...) de que la universidades podian ser útiles, particularmente como promotoras de adelantos técnicos, avivó al grito de que debían cambiar con la época. El reconocimiento universal de que la tecnología descansaba en el progreso científico, y que tal progreso requería un alto grado de especialización, estaba forzando la proliferación y la fragmentación de la instrucción y la investigación"8. Nada menos que el mismo Humboldt quiso establecer en la recién nacida Universidad de Berlín, en 1810, un cierto equilibrio entre Wissenschaft y Bildung, entre el saber dirigido al saber y el saber presidido por la preocupación de su eficacia práctica, si se me permite esta libre interpretación.

En la actualidad, las universidades tienden a convertirse en "multiversidades". En otros términos: no hay acuerdo sobre el sentido de la formación universitaria. A radice, volvemos al conflicto entre las figuras del "hombre cultivado" y el "especialista", que Weber mostrara como un problema central de la teoría educativa.

Pero el desarrollo de tal conflicto muestra por el momento, un claro ganador: como advirtiera Weber, las formas racionales y burocráticas de autoridad y su expresión en lo educativo se cifran en la especialización del científico o del que ejerce una profesión en lo educativo se cifran en la especialización del científico o del que ejerce una profesión liberal. A través de la formación de especialistas, las instituciones educativas asumen la función de control selectivo para la distribución social de oportunidades.

De modo que el sistema educativo se orienta a la producción de especialistas. Nadie se atreverá a discutir algo tan evidente. $Y$ cierto es que el saber se encuentra hoy tan sumamente diferenciado en su estructura misma que no es posible concebir a alguien impuesto en todas las materias del conocimiento.

Pero también es cierto que el sistema genera efectos perversos, para utilizar los términos de Boudon. $Y$ hasta cabe dudar de su eficacia entendida al uso. Asistimos hoy a un retroceso -al parecer imparable-, de las disciplinas fundamentales y a un claro auge de las materias aplicadas. En este sentido, sí que puede hablarse de una cierta homogeneidad del medio universitario, impotente ya para afirmarse en el principio de que no hay ciencias aplicadas sino ciencia que aplicar.

La obsesión por formar profesionales (que no satisface tampoco al mundo empresarial) y el saber organizado con mentalidad minifundista generan no pocos problemas de sentido al interno de la institución académica y obstaculizan gravemente el proceso de avance de los conocimientos. A título de simple muestra de lo afirmado habrá que cuestionarse el hecho, tantas veces comprobado, de que la inmersión en los saberes especializados permita a sus detentadores escapar a la controversia interdisciplinar e incluso a la simple relación diálogica de carácter intelectual, refugiándose en el coto privado y privativo de su especializado saber. Hace ya bastantes décadas, el gran Jaspers advertía al respecto: "La conducta de los miembros de una Facultad ha sido comparada con la de los monos en las palmeras de la arboleda sagrada Benarés: sobre cada palmera hay un mono, y todos parecen estar en paz ocupándose cada uno de sus propias cosas, pero en el momento que cualquiera trata de subirse al árbol de otro se encuentra con una descarga cerrada de cocos"9.

Y respecto de la pretensión de nutrir el mercado de trabajo "haciendo" buenos profesionales a través de la enseñanza especializada, "útil", tampoco podemos sentirnos precisamente satisfechos con el papel de la universidad. Así cito literalmente algunos fragmentos de la crónica aparecida en Diario 16 de 2 de diciembre de 1992, a propósito de Congreso denominado "La reforma de las titulaciones universitarias y su incidencia en el mundo de la empresa", organizado y patrocinado por el Consejo de Universidades y la Fundación Universidad-Empresa: "Los ponentes se mostraron partidarios de una formación amplia y flexible, y en contra de la 'peligrosa' especialización". "La Universidad no debe 'formar profesionales', sino personas con la cabeza flexible y bien preparada, que sean capaces de formarse 
profesionalmente en la empresa". Y transcribo los pasajes más significativos de la intervención de don Clemente Rodríguez Navarro, entonces director de recursos humanos de ATEINSA: "En USA las empresas buscan no a especialistas, sino a personas que puedan integrar una serie de informaciones diversas, extraer sus propias conclusiones y planificar de un modo genérico" y apostaba este profesional por "enseñar a los alumnos a pensar y trabajar ellos mismos, en vez de embutirles en la cabeza unos conocimientos que se les quedarán enseguida obsoletos. En este sentido, dudo de la eficacia de la reforma universitaria".

No podría ser de otra manera. El crecimiento y la competitividad de las empresas, en un contexto de cambios económicos, sociales y técnicos que se desarrollan a toda velocidad, exigen cualidades que no pueden ser ya evaluadas en términos de saberes específicos adquiridos, sino más bien desde la óptica de las capacidades intelectuales, de las actitudes y de los comportamientos, esto es, saber identificar el saber idóneo para cubrir cualquier supuesto, idoneidad para contemplar en paisaje empresarial en clave panorámica o en primer plano, espíritu de creatividad e innovación, facilidad para establecer redes fluidas de comunicación dentro y fuera de la empresa, etc. De modo que lo que la empresa espera de las instituciones educativas es una formación sólida que lleva a los futuros profesionales a la asunción de determinadas actitudes y a la potenciación de los talentos. La competencia específica para desarroIlar la actividad profesional es asunto de las empresas. El principio que fundamenta esta posición es sencillo: una persona que posea las cualidades apuntadas podrá ejercer, al menos en principio, con eficacia y eficiencia, casi cualquier ocupación con un mínimo de aprendizaje ad hoc. Éste ha de ser el mejor servicio de la universidad al mundo laboral. Pretender que la institución universitaria prepare a personas profesionales y competentes para el desempeño de determinadas ocupaciones implica, dicho con toda claridad, no sólo un atentado contra el principio universitario mismo sino también un flaco favor al ámbito empresarial y a la sociedad entera. En cualquier caso, la academia deberá resolver con decisión aquella contradicción de sentido que tan bien expresara Whitehead: "Hay algo entre los gruesos valores especializados del hombre meramente práctico y los finos valores especializados del meramente estudioso. Ambos hombres han perdido algo; y si se suman en uno de los dos juegos de valores, no se obtienen los elementos ausentes"10.
La predicción de Parsons, el gran maestro del estructuralfuncionalismo americano, ha cobrado vida: precisamente la competencia de los técnicos se revela como el gran factor legitimador del orden social actual y provee valiosamente el humus del consenso social. Claro que otros grandes pensadores se adelantaron a ese anuncio (por todos, Scheler y los frankfortianos). En realidad, la clave de la cuestión viene suministrada por aquella distinción weberiana entre Zwerkrationalität y la Wertrationalität, esto es, la razón utilitarista con arreglo a fines y la axiológica. Se trata del dominio de la primera sobre la segunda. Y en conexión con la conciencia cosificada debe tratarse también la relación del saber con la técnica.

Los saberes surgen por necesidades e intereses. Estos "intereses constitutivos de saberes"... "son presupuestos en cualquier acto cognoscitivo y por tanto constituyen los modos posibles de pensamiento, por medio de los cuales puede ser constituida la realidad y se actúa sobre ella"11. Por su parte, el sistema técnico va más allá, claro está, del campo de las actividades de producción de bienes materiales. Según Ellul, la técnica conforma todo un sistema de control social, presenta funciones ocultas y desarrolla una historia latente que es necesario poner en evidencia. Pero, ¿qué entender por "técnica"? La definición de Weber no ha sido superada: "Técnica de una acción significa el conjunto de los medios aplicados en ella, en contraposición al sentido o fin por el que (en concreto) se orienta". Y añade el gran sociólogo alemán: "Técnica racional significa una aplicación de medios que conscientemente y con arreglo a plan está orientada por la experiencia y la reflexión, y en su óptimo de racionalidad por el pensamiento científico. Lo que se entiende concretamente por 'técnica' es fluido: el sentido último de una acción concreta, considerada dentro de la conexión total de una actividad, puede tener carácter de arte 'técnico', o sea, ser medio e instrumento para aqueIla actividad total; sin embargo, con respecto a la acción concreta esa aportación técnica (desde la perspectiva de la actividad total) constituye su verdadero 'sentido' y los medios que aplica son su 'técnica'"12. El sistema técnico es, pues, un sistema de medios. Ahora bien, en la sociedad de nuestros días, el sistema técnico ejerce un dominio total.

Puede hablarse de un dominio total de la técnica "cuando cada aspecto de la vida humana está sometido al control y a la manipulación, a la experimentación y a la observación, de manera que puede lograr para todo una eficacia 
demostrable"13. Así, puede sostener Ellul no sólo que los técnicos forman sistema, sino que, mejor, nuestra sociedad es el sistema técnico. "Bien es verdad que la educación, la política son también técnicas; pero conviene recuperar la distinción entre las técnicas primitivas y pragmáticas y el fenómeno técnico: lo nuevo es el hecho del cálculo, de la sistematización y de la consistencia; las técnicas que usurpan el espacio de la acción espontánea son deliberadas, aplicadas en tanto que técnicas, y es esto lo que rompe el antiguo orden relacional"14. Hasta el extremo de que el sistema técnico determina todos los demás sistemas y se desarrolla imperturbable, irreversible, irremisiblemente, según su propia lógica interna y sin referencia a ninguna finalidad de "vida buena", se retroalimenta y es sustancialmente opresor y dominante.

Es preciso desmentir cuanto antes el entendido común de que la ciencia y la técnica viven unidas por un cordón umbilical. Ni siquiera existe un flujo continuo y directo. La ciencia es para la técnica una memoria y una inteligencia de las que nutrirse. Sin embargo, la situación actual va recordando más y más, al respecto, a la metáfora freudiana de la muerte del padre. El sistema técnico es determinante y totalitario.

En función de los paradigmas dominantes al interno del sistema técnico se va configurando una cultura, pero también una ideología, -"técnicas", si se me permite decirlo así-, congruentes y funcionales según aquellos principios. Tal cultura es -no podría ser otra cosa- de tipo determinista -la eficacia, la eficiencia...- inmediatas y positivas. Tal ideología se funde en la objetividad y la neutralidad, categorias que únicamente compartiría con la ciencia. Nada de proposiciones normativas, pues. Los juicios de valor no encuentran sitio en la técnica: es un principio constitutivo. La técnica es positiva en sí y para sí: es universal. Con lo que su extendida lógica -no por ello, ciertamente, menos extraña- no deja resquicio al juicio axiológico sobre su presencia $y$, en cambio, se asegura un universal juicio afirmativo sobre su naturaleza.

No es que la dominación de la racionalidad técnica haya de conducirlos fatalmente a un "sin sentido bárbaro", como sentenciaron Adorno y Horkheimer. Ni que estén haciendo buenas aquellas palabras de Paul Valéry: que la inhumanidad tiene un futuro grandioso. Pero sería, cuando menos, una ingenuidad pensar que de los juicios de hecho de la ra- cionalidad técnica pudieran colegiarse valores o fines prácticos de praxis como phrónesis. Al respecto, nos advierte Habermas: "... nuevas oleadas de saber técnico irrumpen cada día en la vida social cogiéndola desprevenida, y los nuevos potenciales de un poder ampliado de disposición técnica hacen cada día más manifiesta la desproporción existente entre los resultados de una racionalidad técnica al máximo de su tensión productiva y unos fines perseguidos sin reflexión, unos sistemas de valores esclerotizados y unas ideologías caducas" 15 . Se rinde un culto a la técnica que encuentra su eco en el prestigio social del hombre activo, un hombre que puede hacer cosas. Como dice Gouldner, "la superioridad del técnico sólo es una superioridad de eficiencia". Por ello, "... debemos juzgarlo sólo sobre bases técnicas, y al hacerlo debemos preferir una vida anónica a una vida moral"16. El diagnóstico de Adorno, no por radical deja de ser concluyente: "Un mundo como el de hoy, en el que la técnica ocupa una posición clave, produce hombres tecnológicos acordes con ella. Esto tiene su buena dosis de racionalidad: serán más competentes en su estrecho campo, y este hecho tiene consecuencias en una esfera mucho más amplia. Por otro lado, en la relación actual con la técnica hay algo excesivo, irracional, patógeno. Ese algo está vinculado con el 'velo tecnológico'. Los hombres tienden a tomar la técnica por la cosa misma, a considerarla un fin autónomo, una fuerza con ser propio, y por eso, a olvidar que es la prolongación del brazo humano. Los medios -y la técnica es un conjunto de medios para la autoconservación- son fetichizados porque los fines - una vida humana digna- han sido velados y expulsados de la conciencia de los hombres"17. Es inevitable: la razón dominada por la técnica degenera en pensamientos y acciones conformistas y devalúa el mundo de los afectos. El nuevo demiurgo, la "objetividad", legitima ese nuevo ser-en-el-mundo. En la racionalidad técnica no caben las categorias éticas; no hay el menor resquicio, pues, tampoco para la crítica.

La objetividad científica se manifiesta, dice Weber, en el cálculo y comparación de los medios que permiten el logro de un fin. La técnica no es un saber objetivo, a no ser que recluyamos el concepto nudo de objetividad en el reducto interesado de las opciones metodológicas de corte positivista. El saber técnico es parcial, y sus pretensiones holistas revisten una especial gravedad en tanto se afirma aquél como verdad universal, como concepción general sobre la sociedad y sobre los hombres. Sin embargo, esos esquemas de legitimación de carácter técnico -y de base 
jurídico-positiva- se empeñan en presumirse instalados en la certeza y la objetividad.

La preeminencia técnica inaugura un tipo especial de control social por el que los demás conocimientos van excluyéndose de la categorización de útiles, prácticos, buenos, sabios $u$ objetivos. Tal posición preeminente levanta un escenario intelectual en el que lo técnico se reserva un papel protagonista que necesita el refuerzo de efectos especiales tendentes al arrumbamiento de los saberes abstractos, sobre todo de los humanistas, tachados a menudo de ideológicos o axiológicos. Esa racionalidad técnica reconoce, ante todo, el valor de la eficacia. En realidad, como ya ha quedado dicho, estamos ante una cultura y una ideología que confina el mundo de las relaciones sociales -y preferentemente, el conocimiento-, en la cárcel de la esfera utilitarista, de la lógica productiva. La universidad no podría librarse de las acometidas de esa potente racionalidad $y$, desde luego, no lo ha hecho. De modo que estamos ante una universidad colonizada por esa racionalidad técnica.

Veblen ya advirtió sobre las nefastas consecuencias de "dirección de las universidades por hombres de negocios"18. Sin tomar las aguas tan arriba, si que puede afirmarse, con todo, que "la intelectualidad humanística", empleando los términos de Gouldner, declina "en influencia relativa dentro de la proliferación de enseñanzas técnicas en la universidad, con lo cual se hace más vulnerable a la alienación"19. De manera más clara, dicho sea en palabras de Drucker: "Existe el riesgo de producir una sociedad de bárbaros escolarizados"20.

El dominio de la racionalidad técnica al interno de la institución académica desempeña un papel alienante en la medida, a nuestros efectos, en que relega a un segundo plano a otras esferas del conocimiento. Como dice Habermas: "El rendimiento peculiar de esta ideología consiste en que disocia la autocomprensión de la sociedad del sistema de referencia de la acción comunicativa y de los conceptos de la interacción simbólicamente mediada y los sustituye por un modelo científico. En la misma medida, la autocomprensión culturalmente determinada de un mundo social de la vida queda sustituida por la autocosificación de los hombres bajo las categorias de la acción racional con respecto a fines y del comportamiento adaptativos"21. Del mismo modo que la universidad se muestra socialmente escindida, se ha diluido en sus concepciones epistemológicas y normativas.

La educación universitaria aparece, en definitiva, presidida por el designio del desprecio que a los ojos de la lógica productiva merece el saber abstracto, al que no se ve capaz de transformarse, al contrario que el saber técnico, en fuerza productiva. De manera que lo que hacemos en la universidad es, más bien, alimentar un proceso de reproducción social y no una acción confirmativa que llevara en sí misma el germen del cambio social hacia la libertad y la plena realización de las personas. La universidad puede y debe contribuir decisivamente a la reserva de autonomía de las personas resistiéndose a esa racionalidad que el poder-legitimado en parte por la opinión pública- pretende imponer. Es necesario que la universidad se comprometa a asegurar la formación integral. Ahora bien, si la universidad rechaza la llamada a preparar técnicos, no sobrevivirá. Pero si rechaza el culto a los valores "no útiles", dejará de merecer el título de universidad. Hace falta un puente entre el discurso de los técnicos y el de los humanistas. Y éste puede tenderse con el reconocimiento de que el desarrollo tecnológico o la coordinación de trabajos parcelarios construyen procesos que necesitan una autoridad, que a su vez, puede encontrar sus fuentes en el saber fundamentado y la experiencia como luces que nos hagan ver y nos guien hacia la recta acción. En definitiva, la verdadera cultura a la que la universidad debe aspirar sólo puede consistir en la correlación de todas las disciplinas en una formación total que englobe también a la persona moral.

\section{La defensa y la universidad}

El mundo de la defensa en España, ha estado muy alejado de la universidad, durante muchos años. Es cierto, que en algún momento se ha querido dar protagonismo a las cuestiones de la defensa desde materias como las relaciones internacionales, pero de una forma muy superficial y yo diría, bastante confusa.

Es cierto, que desde alguna universidad, se quiso dar carta de naturaleza a las cuestiones relacionadas con la defensa a través de la creación de Cátedras de Estudios, pero éstas tardaron en cuajar en el seno de las Facultades universitarias.

La creación de las universidades privadas y la puesta en marcha de nuevos planes de estudios, pusieron las bases 
para estructurar como disciplina académica los estudios reglados sobre defensa y seguridad, desde distintas vertientes del saber.

En 1992, la Universidad CEU-San Pablo, inicia su andadura como tal. Atrás queda un bagaje académico universitario como Centro Universitario adscrito a la Universidad Complutense, donde miles de profesionales se han formado en sus aulas a lo largo de más de 50 años, y dando vida a un proyecto que estaba en la mente de su fundador don Ángel Herrera Oria, muchos años antes que éste se hiciera realidad.

De acuerdo con la normativa salida de la creación de las universidades privadas, los nuevos planes de estudios deberian contemplar asignaturas troncales, obligatorias, optativas y de libre configuración.

En este contexto, el Patronato de la Fundación San PabloCEU, acuerda en entre otras decisiones, de que se incorpore una materia en la Facultad de Humanidades y Ciencias de la Comunicación que estudie la seguridad y la defensa, desde la perspectiva de la identidad y la cultura de la defensa con el fin de que los alumnos asuman lo que se empezaba a llamar "conciencia de defensa", como algo que afecta a todos los ciudadanos y no es exclusivo de aquellos que pertenecen a las Fuerzas Armadas.

La asignatura, se denomina "Información sobre Defensa y Seguridad", nombre con que sigue impartiéndose en la actualidad, y que desde sus inicios se encuadró dentro de las asignaturas optativas, semestrales, para alumnos de segundo ciclo de Periodismo, Comunicación Audiovisual y Publicidad.

Este nuevo reto, fue seguido con cierta expectación, no solamente por tratarse de una materia que en la Universidad era nueva, sino también porque sale a la luz en un momento no precisamente el más apropiado: la insumisión y la objeción de conciencia, estaban todos los días en los medios de comunicación, informando sobre hechos de esta naturaleza. No debemos olvidar que todavía esta vigente el Servicio Militar Obligatorio, y que los alumnos de cuarto y quinto de carrera estaban en "edad militar, por lo que la sensibilidad de estas cuestiones era mayor".

En paralelo a la asignatura reglada, se pusieron en marcha seminarios y cursos de verano relacionados con te- mas de defensa, que tuvieron desde un principio una buena cogida, pues se incorporaban a las aulas personas ajenas a la universidad, y por otro lado, se volvian a ver uniformes militares en las aulas. Nos encontrábamos en plena operación de imagen, por un lado y por otro que los alumnos vean, hablen y pregunten sobre temas de defensa a los protagonistas de la misma, es decir a los militares.

Con ello, quisimos aportar nuestro grano de arena, y romper el hielo que existía entre sociedad civil y militar.

A medida que se ha ido consolidando esta situación, se hacía necesario una cooperación más estrecha con el Ministerio de Defensa, y más concretamente con el Instituto Español de Estudios Estratégicos, que siempre ha brindado su colaboración y ayuda en todo lo relacionado con cursos y seminarios.

Y precisamente, en ese espíritu de colaboración y con el fin de dar carta de naturaleza a todas las actividades relacionadas con la defensa, se acuerda la creación de una Cátedra de Estudios sobre Defensa y Seguridad, cuyo fin principal es la de acoger todas las actividades académicas, de investigación, cursos y seminarios dirigidos a fomentar la cultura de defensa entre los alumnos universitarios.

Y así en el año 2000, ve la luz la Cátedra de Estudios sobre Defensa y Seguridad "Abelardo Algora", en homenaje a quien fue presidente de la Fundación Universitaria San Pablo-CEU, impulsor de su universidad, y coronel del Cuerpo Jurídico del Ejército de Tierra. Cátedra que hoy desarrolla una parte de sus actividades con el Centro Superior de Estudios de la Defensa Nacional (CESEDEN), a través de la Escuela de Altos Estudios de la Defensa, y con el Instituto Español de Estudios Estratégicos.

Sin duda, esta simbiosis Universidad y Fuerzas Armadas, a través de estos organismos, ha sido de gran acierto para acercar más al estudiante universitario al mundo de la defensa, que representa y sobre todo llegar a la conciencia de que la defensa somos todos.

De cualquier forma, y con la vista atrás, podemos afirmar que el riesgo que asumimos en su momento mereció la pena, y aunque despacio, vamos viendo sus frutos.

ARBOR CLXXXIV Anejo 2 [2008] 89-100 ISSN: 0210-1963

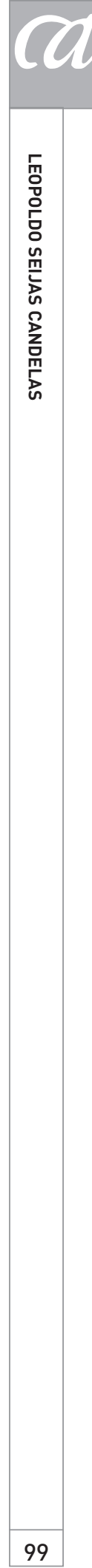




\section{NOTAS}

1 Emplearé el término "educación" siguiendo el recordatorio de Mialaret en tres sentidos: para designar un sistema; para indicar el resultado de un proceso; y para indicar, como tal, el proceso en sí mismo considerado. Ahora bien, según las necesidades del discurso recurriré a términos tales como instrucción, formación, cualificación, competencia o profesionalismo, que presentan significaciones distintas bien que cercanas al concepto central.

Asimismo, quiero señalar que entiendo el término "multiversidad" en el sentido otorgado por Clark Kerr, el primero en utilizarlo. Vide, al respecto, su escrito The uses of University, Cambridge, Mass., Harvard University Press, 1973.

2 Vallespin, F., "Universidad/Sociedad", en Claves de razón práctica, n. 6 , octubre 1990, pp. 18-24, p. 20. Vide también: L'espace éducatif européen, de Andrieu, J., Conseil économique et social, junio 1992. Además Ballion, R., La Bonne École, Hatier, 1981, y el Dossier de Le Monde de l'éducation, de octubre 1991.

3 García Gual, C., "Sobre la degradación de la educación universitaria", Claves de la razón práctica, n. ${ }^{\circ} 2$, mayo 1990, pp. 52-57.

4 Parsons T., en Halsey, A. H.; Floud, J., y Anderson, C. A. (eds.), Education, Economy and Society, New York, Free Press, 1961, pp. 434-455.

5 Vallespín, F., ibid.
6 Citado por A. F. Utz, Ética social, III, Herder, Barna, 1988, 330 pp., p. 148.

7 Habermas, J., Teoría y praxis. Estudio de filosofía anual, Ed. Tecnos, Madrid, 1987, p. 34.

8 Hutchins, R. M., Aprendizaje y Sociedad, Monte Ávila, Caracas, 1969, 149 pp., p. 125.

9 Hutchins, R. M., ob. cit., p. 128.

10 Citado por E. Ashby, La Tecnología y los Académicos, Monte Ávila, Caracas, 1969, 166 pp., p. 147.

11 Carr, W. y Kemmis, S., Teoría crítica de la enseñanza, Martínez Roca, 1988, Barna, 245 pp., p. 147.

12 Weber, M., Economía y sociedad, FCE, México, 1983, 1.237 pp., p. 47.

13 Ellul, J., Le Système technicien, París, Calmann-Lévy, 1977, p. 94.

14 Ellul, J., ob cit., p. 278.

15 Habermas, J., Ciencia y técnica como "ideología", Tecnos, Madrid, 1986, 181 pp., p. 147.

16 Gouldner, A. W., La dialéctica de la ideología y la tecnología, Alianza, Madrid, 1978, 372 pp., p. 236.

17 Adorno, T. W., La educación de Auschwitz, conferencia publicada en Zum Bildungsbegriff des Gegenwart, Frankfurt, 1967, pp. 111 y ss. Traducción española en Consignas, Amorrortu, pp. 80-95, p. 91.

18 Veblen, T., The Higher Learning in America, New York, B. W. Huebsch, 1918, pp. 164 y ss.

19 Gouldner, A. W., ob. cit., p. 241.

20 Drucker, P., Las nuevas realidades, Edhasa, Barna, 1989, 365 pp., p. 354.

21 Habermas, J., Ciencia y técnica como idelogía, p. 89.
Recibido: 22 de enero de 2008 Aceptado: 26 de marzo de 2008 